\title{
Interactive Haptic Transmission for Remote Control Systems
}

\author{
Toshio Asano Yutaka Ishibashi Seiji Kameyama \\ Department of Computer Science and Engineering, Graduate School of Engineering, \\ Nagoya Institute of Technology, Nagoya, 466-8555 Japan \\ \{asano, ishibasi, kame_oji\}@mcl.elcom.nitech.ac.jp
}

\begin{abstract}
In this paper, we propose a remote control system which controls a haptic interface device with another remote haptic interface device. Haptic feeling can be transmitted by the system. Applications of the system are a remote calligraphy system, a remote drawing instruction system, a remote medical operation system, and so on. The paper deals with the remote drawing instruction system by which an instructor trains a learner how to draw pictures or figures while conveying the sense of force through the Internet. In order to clarify what kinds of control are needed in the system, we examine the influences of network delay, delay jitter and packet loss on the output quality of haptic media by subjective assessment of drawing figures.
\end{abstract}

\section{INTRODUCTION}

Applications with haptic media in distributed virtual environments based on computer graphics (CG) have been actively researched. For example, there are a remote operation system using a master-slave robot in the medical field [1], [2] and a remote control system in a place of strong radioactivity in the robot control field [3]. Production and appreciation of contents in various types of museums are also proposed in artistic and educational fields [4], [5]. This paper proposes a remote drawing instruction system using haptic interface devices in the educational field. The system transmits the haptic feeling of an instructor and a learner as haptic media streams interactively; therefore, the educational effect can be improved greatly.

When a haptic media stream is transferred over the Internet, the output quality of the media stream may deteriorate significantly owing to the network delay, delay jitter and packet loss. Requirements for the output quality of haptic media are much more stringent than those of audio and video [6], [7]. Therefore, it is important to investigate the influences of the network delay, delay jitter and packet loss on the output quality of haptic media. In this paper, we assess the influences subjectively, and we clarify what kinds of control are required for the remote drawing instruction system.
The rest of this paper is organized as follows. Section 2 outlines the remote drawing instruction system. Section 3 describes the experimental system. Section 4 explains the subjective assessment method, and experimental results are presented in Section 5. Section 6 concludes the paper.

\section{REMOTE DRAWING INSTRUCTION SYSTEM}

\subsection{System configuration}

The remote drawing instruction system enables navigation of brush stroke while an instructor and a learner feel the sense of force interactively. The outline of the system is shown in Fig. 1. Each terminal of the two users (i.e., the instructor and the learner) is connected to PHANToM Omni (just called PHANToM here) [8] as a haptic interface device.

The instructor draws a figure on the canvas composed by CG (computer graphics) with PHANToM, which is considered as a paintbrush. The position information of PHANToM in the virtual space is transmitted between the instructor's terminal and the learner's terminal. At the learner's terminal, the force applied to his/her PHANToM is calculated based on the received position information of the instructor's PHANToM and the position information of the learner's PHANToM. Then, the figure is drawn on the canvas. By using the system, the brush stroke of the instructor and that of the learner are shared by both instructor and learner.

The functions implemented at each terminal are 1) input of PHANToM position of the terminal in the virtual space, 2) transmission of position information to the other terminal, 3) reception of PHANToM position of the other terminal and media synchronization control, 4) calculation and output of the force based on the position comparison between the instructor's PHANToM and the learner's PHANToM, and 5) figure drawing and position update of the two PHANToMs, as shown in Fig. 2.

The transmission rate of position information and the rendering rate are $30 \mathrm{~Hz}$ at each terminal. The frequency of the servo loop for input and output of PHANToM position information is $1000 \mathrm{~Hz}$ [9]. 


\subsection{Drawing mechanism}

The instructor's terminal and the learner's terminal draw a fixed-size square at each PHANToM position on the canvas every 33 milliseconds. Consecutively-drawn two squares are connected with a line whose thickness is equal to one side of the squares as shown in Fig. 3 (a). Each learner's line is slightly shifted from the instructor's corresponding line in the figure for ease of viewing.

When position information is lost in the Internet (see Fig. 3 (b)), the drawn line is completed with the square drawn by the most recently-received position information. In Fig. 3 (b), the instructor's packet with sequence number 3 is lost. In this case, different force from the original force is applied to the learner. Loss of position information can be judged by the sequence number given to position information.

\subsection{Method of transmitting force}

The method to transmit the instructor's force, which controls the remote learner, is shown in Fig. 4, where force $\boldsymbol{F}(t)$ is applied to the learner's PHANToM at $t$ seconds $(t \geq$ $0) . \boldsymbol{F}(t)$ is calculated as follows. Let the sequence numbers which are given to the latest position information of the instructor's PHANToM and that of the learner's PHANToM at $t$ seconds be denoted by $i$ and $j(i \geq 1, j \geq 1)$, respectively. In Fig. 4, we set $i=3$ and $j=4$. We also denote the position vector of the instructor's PHANToM by $\boldsymbol{T}_{i}\left(x_{i}, y_{i}, z_{i}\right)$, and that of the learner's PHANToM by $\boldsymbol{S}_{j}\left(x_{j}, y_{j}, z_{j}\right)$. The force $\boldsymbol{F}(t)$ is given by $\boldsymbol{F}(t)=k \boldsymbol{V}_{i j}$, where $k$ and $\boldsymbol{V}_{\boldsymbol{i j}}$ are the spring coefficient and the difference vector, respectively, and we have the following relation: $\boldsymbol{V}_{i j}=\boldsymbol{T}_{i}\left(x_{i}, y_{i}, z_{i}\right)-$ $\boldsymbol{S}_{j}\left(x_{j}, y_{j}, z_{j}\right)$. That is, the learner's PHANToM is pulled by the force $\boldsymbol{F}(t)$ so that the position of the learner's PHANToM approaches that of the instructor's PHANToM on the canvas. The instructor's PHANToM is controlled similarly.

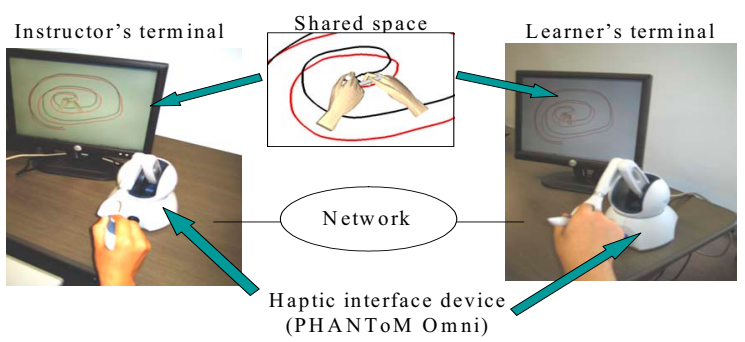

Fig. 1. Remote drawing instruction system.

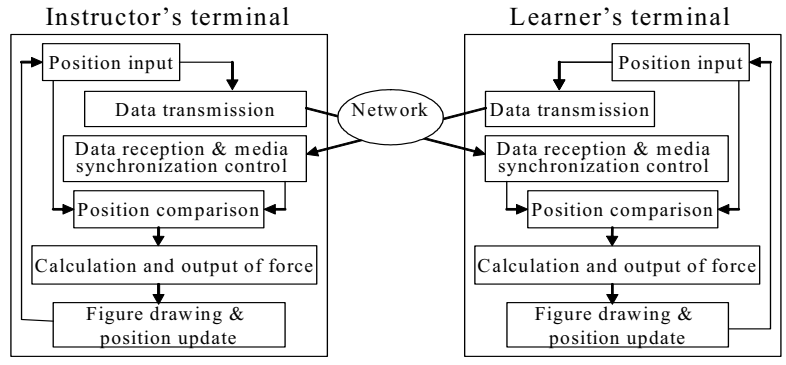

Fig. 2. Functions of remote drawing instruction system.

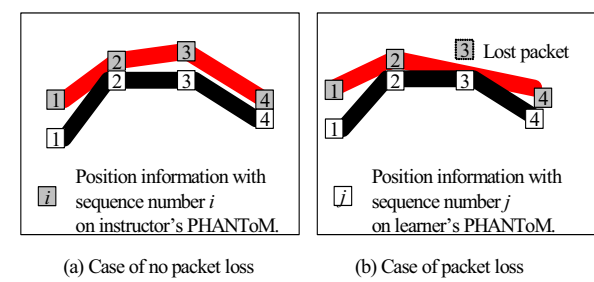

Fig. 3. Drawing mechanism.

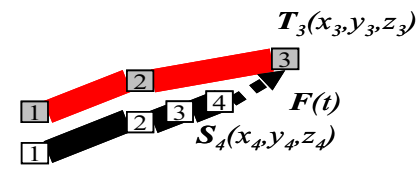

Fig. 4. Method of transmitting force.

\section{EXPERIMENTAL SYSTEM}

The instructor's terminal is connected to the learner's terminal through a network emulator (NIST Net). Each terminal sends/receives 328 bytes of data that include the sequence number and position information as a media unit $(M U)$, which is an information unit for media synchronization [10]. By using NIST Net, we generate a constant delay or delay jitter and packet loss for each MU transmitted between the instructor's terminal and the learner's terminal. In addition, the MUs are transmitted by UDP.

For media synchronization control at each terminal, we adopt Skipping [10]. Skipping outputs only the most recently-arrived MU every millisecond. Obsolete MUs are skipped. We do not absorb network delay jitter in order to examine the influence of network delay jitter in Section 5. Thus, we employ Skipping in this paper.

\section{SUBJECTIVE ASSESSMENT METHOD}

In the subjective assessment, fourteen subjects whose ages were between 21 and 24 drew the following three kinds of figures: "Whorl," "star" and "saw" (see Fig. 5). To investigate how the difference in brush stroke influences the subjective assessment, "whorl," "star" and "saw" figures were selected as brush strokes with rotation, turns and sharp turns, respectively. We adopted the single stimulus method in ITU-R BT.500-10 [11], which is a recommendation for subjective assessment of television pictures, because there is no standard for subjective assessment of haptic media. After practicing, each subject gave a score based on Table 1 according to the degree of degradation of haptic feeling for each stimulus.

We carried out experiments of constant delay and delay jitter. In the first experiment, we selected an additional constant delay of $0,100,200,300$, or $400 \mathrm{~ms}$ in a random sequence for each figure and each subject. After the learner assessment, the instructor assessment was carried out. The rate of packet loss was $0 \%$ in this case. 
An example of drawn "whorl" at the learner's terminal is shown in Fig. 6, where the learner's brush stroke is not smooth and diverges from the instructor's brush-stroke largely when the additional constant delay is $100 \mathrm{~ms}$. When the haptic feeling deteriorates, stronger force is necessary to operate the PHANToM; without the strong force, smooth operation is impossible. The instructor and learner feel similar degradation.

In the experiment of delay jitter, the assessment was carried out by producing additional delays according to the Pareto normal distribution. We set the average additional delay to $100 \mathrm{~ms}$ or $200 \mathrm{~ms}$. In the case of $100 \mathrm{~ms}$, the standard deviation of the additional delay was selected from among 0,20 , and $40 \mathrm{~ms}$. In the case of $200 \mathrm{~ms}$, we selected the standard deviation from among 0, 20, 40, and $60 \mathrm{~ms}$.

The number of stimuli per subject in the network delay experiment and that in the delay jitter experiment were 30 and 42 , respectively. The total time per subject was between 15 and 20 minutes.

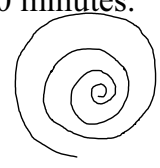

(a) whorl

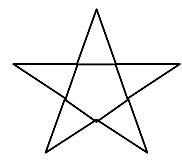

(b) star

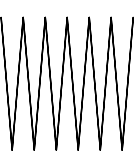

(c) saw
Fig. 5. Three kinds of figures drawn in experiment.

Table. 1. Five-grade impairment scale.

\begin{tabular}{|c|c|}
\hline score & description \\
\hline \hline 5 & imperceptible \\
\hline 4 & perceptible, but not annoying \\
\hline 3 & slightly annoying \\
\hline 2 & annoying \\
\hline 1 & very annoying \\
\hline
\end{tabular}

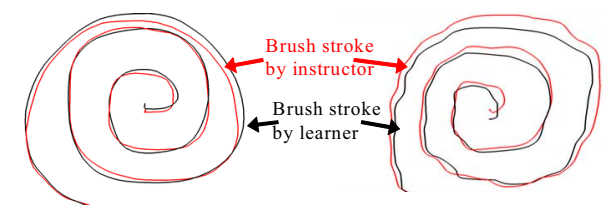

(a) Additional constant delay: $0 \mathrm{~ms}$ (b) Additional constant delay: $100 \mathrm{~ms}$

Fig. 6. Example of drawn "whorl" at the learner's terminal.

\section{EXPERIMENTAL RESULTS}

\subsection{Influence of network delay}

We show the Mean Opinion Score (MOS) values [11] at the instructor's and learner's terminals as a function of the additional constant delay in Figs. 7 and 8, respectively. In Fig. 7, the MOS at the instructor's terminal is larger than or equal to approximately 3 when the additional constant delay is smaller than or equal to around $200 \mathrm{~ms}$. When the additional constant delay is larger than or equal to around $300 \mathrm{~ms}$, the MOS is smaller than or equal to about 2. In this area, it was difficult for the instructor to draw the figures since strong force was necessary to operate the PHANToM. On the other hand, in Fig. 8, the MOS at the learner's terminal is larger than or equal to about 3 when the additional constant delay is smaller than or equal to around $300 \mathrm{~ms}$. When the additional constant delay is $400 \mathrm{~ms}$, the MOS is smaller than 3.

Furthermore, we see in Figs. 7 and 8 that the MOS tends to become larger in the order of "whorl," "star" and "saw." Therefore, we claim that "saw" is more influenced by network delay than the other brush strokes. The reason is that larger force is necessary for a turn when position information arrives late. However, the differences in MOS among the three figures are small.

From the above observations, we claim that the influence of network delay on the MOS at the instructor's terminal is larger than that at the learner's terminal. The reason is that the learner regards the degradation caused by the additional delay as the force exerted by the instructor. That is, he/she may think that there is no influence of network delay. Also, as the network delay becomes larger, larger force is necessary for the instructor to operate the PHANToM. Therefore, we need adaptive force control [12] which dynamically changes the force by modifying the value of the spring coefficient according to the network load.

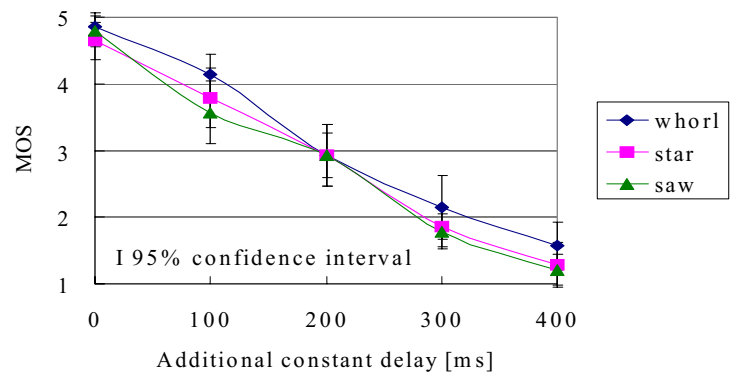

Fig. 7. MOS versus additional constant delay at the instructor's terminal.

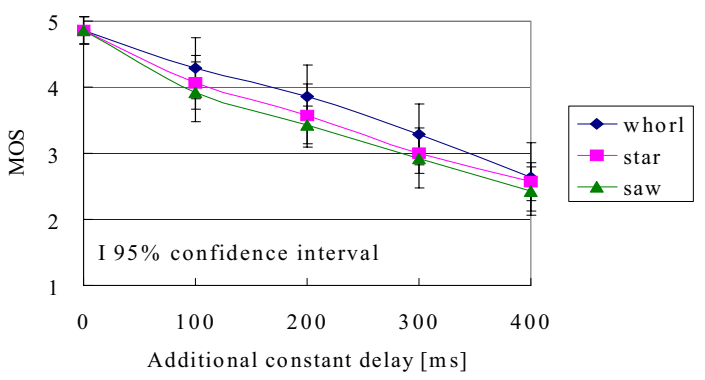

Fig. 8. MOS versus additional constant delay at the learner's terminal.

\subsection{Influence of delay jitter}

In the delay jitter experiment, the MOS values at the instructor's and learner's terminals are shown in Figs. 9 and 10 , respectively. In the figures, we see that the MOS at the learner's terminal is larger than that at the instructor's terminal when the standard deviation of the additional delay is $0 \mathrm{~ms}$, as in the previous subsection. However, when the 
standard deviation is larger than or equal to about $40 \mathrm{~ms}$, the MOS at the learner's terminal is almost the same as the MOS at the instructor's terminal. Moreover, the MOS decreases as the standard deviation increases. Therefore, media synchronization control (except Skipping) to absorb network delay jitter is necessary.

In Figs. 9 and 10, the MOS tends to increase in the order of "whorl," "star" and "saw." This is the same trend as that in Figs. 7 and 8. Skipping causes loss of position information in the case where there is delay jitter [10]. The algorithm outputs only the most recently-arrived MU every millisecond. Obsolete MUs are skipped. Therefore, smooth control becomes more difficult as delay jitter becomes larger.

We also performed the packet loss experiment. As a result, we found that prediction control and error control are necessary. We are currently implementing adaptive force control, media synchronization control, prediction control, and error control.
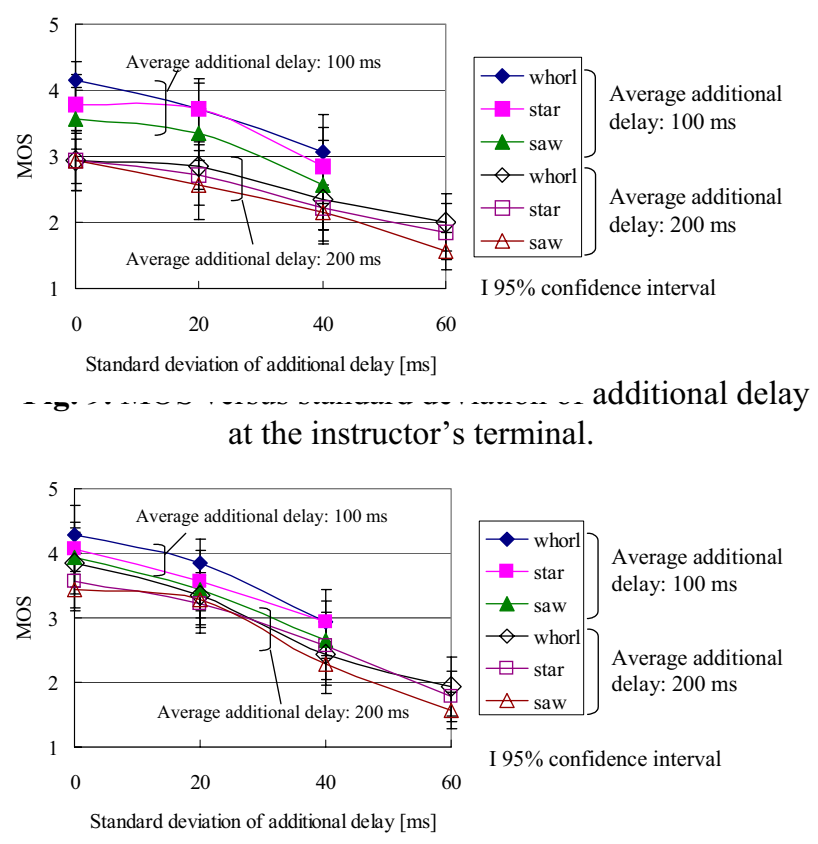

Fig. 10. MOS versus standard deviation of additional delay at the learner's terminal.

\section{CONCLUSIONS}

Influences of network delay, delay jitter and packet loss on the output quality of haptic media in a remote drawing instruction system were investigated by subjective assessment in this paper. As a result, we found that the influence at the instructor's terminal is greater than that the learner's terminal in the case of network delay. Subjects acting as an instructor felt slightly annoyed when the additional constant delay is larger than or equal to about 200 ms. Subjects acting as a learner felt slightly annoyed when the additional constant delay is larger than or equal to about $300 \mathrm{~ms}$. The influences of delay jitter on the instructor and learner have almost the same tendency. Moreover, there are small differences in output quality among the drawn figures. We claim that adaptive force control, media synchronization control, prediction control and error control are necessary to attain a higher output quality of haptic media.

In the next step of our research, we plan to perform an experiment using other types of figures in the variety of network environments.

\section{Acknowledgments}

This work was partly supported by the Grant-In-Aid for Scientific Research (C) of Japan Society for the Promotion of Science under Grant 16560331 and the Kayamori Foundation of Informational Science Advancement.

\section{REFERENCES}

[1] Intuitive Surgical, Inc., $\mathrm{http}: / /$ www.intusurg.com/html/davinci.html.

[2] A. Liu, F. Tendick, K. Cleary, and C. Kanufmann, "A Survey of Surgical Simulation: Applications, Technology, and Education," Presence, vol. 12, no. 6, pp. 599-614, Dec. 2003.

[3] N. Turro, O. Khatib, and E. Coste-maniere, "Haptically Augmented Teleoperation," in Proc. IEEE International Conference on Robotics and Automation, 2001.

[4] M. C. Lin, W. V. Baxter, V. E. Scheib, and J. D. Wendt, "Physically Based Virtual Painting," Communications of the $A C M$, vol. 47, no. 8, pp. 41-47, Aug. 2004.

[5] T. Asano and Y. Ishibashi, "A Haptic Museum in Distributed Virtual Environments," in Proc. WTC/ISS2004, Sep. 2004.

[6] ITU-T Recommendation G.114, "Transmission Systems and Media, General Characteristics of International Telephone Connections and International Telephone Circuits: One-Way Transmission Time," Feb. 1996.

[7] S. Matsumoto, I. Fukuda, H. Morino, K. Hikichi, K. Sezaki, and Y. Yasuda, "The Influences of Network Issues on Haptic Collaboration in Shared Virtual Environments," in Proc. the Fifth PHANToM Users Group Workshop, Oct. 2000.

[8] J. K. Salisbury and M. A. Srinivasan, "Phantom-Based Haptic Interaction with Virtual Objects," IEEE Computer Graphics and Applications, vol. 17, no. 5, pp. 6-10, Sep./Oct. 1997.

[9] SensAble Technologies, Inc., "3D Touch SDK OpenHaptics Toolkit Programmer's Guide," Version 1.0, 2004.

[10] Y. Ishibashi, S. Tasaka, and T. Hasegawa, "The Virtual-Time Rendering Algorithm for Haptic Media Synchronization in Networked Virtual Environments," in Proc. of the 16th International Workshop on Communications Quality and Reliability (CQR2002), pp. 213-217, May 2002.

[11] ITU-R BT.500-10, Methodology for the subjective assessment of the quality of television pictures," International Telecommunication Union, 2001.

[12] M. Fujimoto and Y. Ishibashi, "A Compensation Scheme for Network Delay Jitter of Haptic Media in Networked Virtual Environments," in Proc. SCI2004, July 2004. 